\title{
Angular Distribution of High Energy Electrons Following Radiation
}

Leonard C. Maximon

LI.S. DEPARTMENT OF COMMERCE

National Bureau of Standards

Center for Radiation Research

Gaithersburg, MD 20899

and

Alfred Lepretre

Centre d'Etudes Nucleaires de Saclay

Division de la Physique

Departement de Physique Nucieaire

Service de la Metrologie et

de la Physique Neutroniques Fondamentales

91131 Gif-sur-Yvette Cedex, France

October 1985

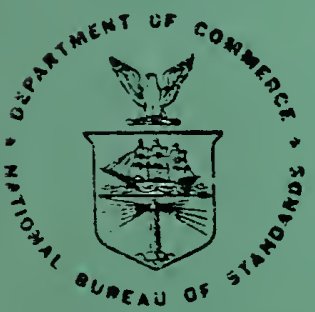

U.S. DEPARTMENT OF COMMERCE

$Q C$
100
$U 56$
$84-2854$
1985
$C .2$

NAL BUREAU OF STANDAROJ 

NBSIR 84.2854

$\ldots$
ANGULAR DISTRIBUTION OF HIGH ENERGY ELECTRONS FOLLOWING RADIATION

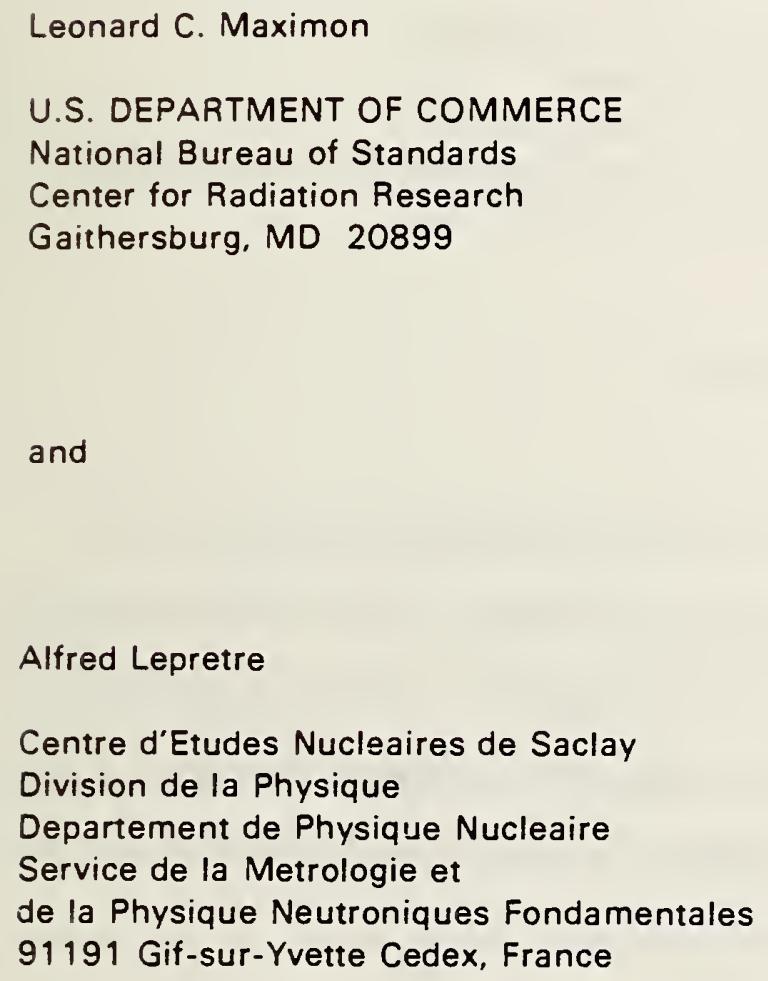

October 1985

U.S. DEPARTMENT OF COMMERCE, Malcolm Baldrige, Secretary NATIONAL BUREAU OF STANDARDS, Ernest Ambler, Director 



\author{
Leonard C. Maximon \\ Center for Radiation Research \\ National Bureau of Standards \\ Gaithersburg, MD 20899
}

\title{
Alfred Leprêtre
}

Centre d'Etudes Nucleaires de Saclay

Division de la Physique

Departement de Physique Nucleaire

Service de la Metrologie et de la Physique Neutroniques Fondamentales

91191 Gif-sur-Yvette Cedex, France

\section{ABSTRACT}

We derive an expression for the angular distribution of high energy electrons which have undergone scattering and radiated a photon, integrated over the directions of the emitted photon, in the region of small scattering angles, for which the atomic form factor must be taken into account but the nuclear structure may be neglected. This

distribution is analogous to Schiff's high-energy small-angle distribution for photons, integrated over the final electron angles. We show that the correction to the energy-angle distribution of electrons due to atomic screeing is identical in form to the correction to the energy-angle distribution of photons. This correction involves an integral over the atomic form factor, and is evaluated in closed form for the Thomas-FermiMoliere model. A very simple expression is obtained for the case of complete screening.

Key words: angular distribution of scattered electrons; atomic screening effects in electron scattering; bremsstrahlung; high energy electron scattering; small angle electron scattering cross section; Schiff energy-angle distribution. 


\section{ACKNOWLEDGMENTS}

We wish to express our gratitude to Gloria Wiersma, to whom we are indebted for the skillful and expeditious typing of this report. 


\section{CONTENTS}

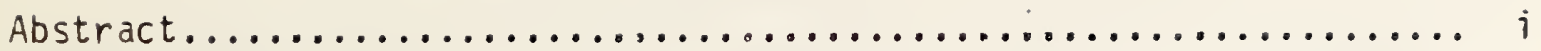

Acknowledgments....................................

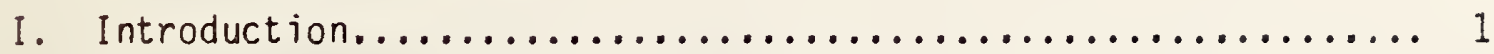

II. The Cross Section Integrated Over Photon Directions......... 3

III. Technical Preliminaries........................... 8

IV. Derivation of the Screening Correction................. 16

V. Screening Correction in the Thomas-Fermi-Moliere Mode1...... 34

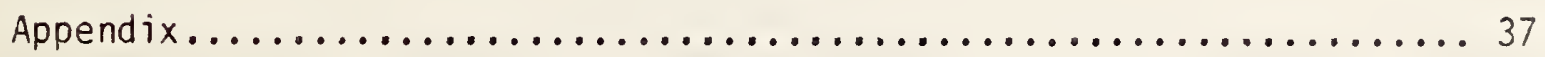

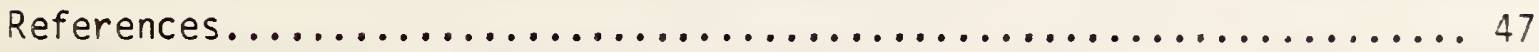



In this note we derive an expression for the angular distribution of high energy electrons which have undergone scattering and radiated a photon, integrated over the directions of the emitted photon. This cross section has been obtained previously under conditions pertinent to large angle, 1 arge momentum transfer electron scattering experiments [1-3]. Here we are concerned with this same cross section, but under conditions appropriate to a photon tagging system, for which the important contribution comes from small angle scatterings, corresponding to momentum transfers $q \lesssim O(m c)$. Although momentum transfers $q>0(m c)$ give a negligible contribution if one subsequently also integrates over the angles of the scattered electron, our angular distribution for the scattered electron is in fact valid for the wider range of momentum transfers, $q_{m} \leqq q \ll \frac{1}{R}$, where $q_{m}$ is the minimum possible momentum transfer and $R$ is the nuclear radius, i.e., it is valid provided that the nuclear form factor does not enter significantiy. We therefore neglect target recoil, and in place of the nuclear form factor which appears as $\mathscr{I}^{2}(q)$ in the completely differential cross section involving large momentum transfers, we now have the atomic form factor, appearing as $[1-F(q)]^{2}$. (See [1], p. 81345, comments following (5).) We start therefore with the Bethe-Heitler (Born approximation) differential cross section for bremsstrahlung. We note that the integration of this cross section over the arigles of the final electron, assuming small momentum transfers, is well-known [4], and in the case of complete screening results in the Schiff energy-angle distribution $[4,5]$. 
The integration of the bremsstrahlung cross section over final electron directions has also been performed [6], including Coulomb corrections, in the region of small momentum transfers $q \lesssim O(m c)$, and some of the analys is given in [6] will be of use to us in the present work.

However, we have not found, in the available literature, an expression for the bremsstrahlung cross section integrated over photon directions that is useful for the region of small momentum transfers (the equivalent of Schiff's expression, but integrated over photon directions rather than final electron directions). This lack is quite understandable. The major interest in the cross section for small angles (low momentum transfers) has been to obtain the angular distribution of bremsstrahlung (integrated over electron directions) since the photons are emitted preferentially in the forward direction. On the other hand, when the integration over photon directions has been performed, it has been in connection with high energy electron scattering experiments, which are performed at large angles and large momentum transfers in order to obtain nuclear structure information. 
We turn now to the Bethe-Heitler (Born approximation) cross section integrated over photon angles, but with no approximations concerning the energies of the incident and final electron or the angles of the scattered electron. This has been given by Maximon and Isabelle [1] in connection with the radiative tail in elastic electron scattering (eqs (4) and (5) on p. B1345 of [1]). We need only replace the nuclear structure function $F(q)$ in eq (4) of $[1]$ by $1-F(q)$, where $F(q)$ is the atomic form factor. We then have*

$$
\frac{d \sigma}{d \Omega}=\frac{1}{2 \pi} \frac{e^{2}}{\hbar c}\left(\frac{Z e^{2}}{m c^{2}}\right)^{2} \frac{p_{2}}{p_{1}} \frac{d \epsilon_{2}}{x} \int_{q_{m}^{2}}^{q_{M}^{2}} \frac{[1-F(q)]^{2}}{q^{4}}\{\} d\left(q^{2}\right)
$$

where

*The most direct way of arriving at the result given in (2) is the procedure used in [3] (rather than that followed in [1]). In [3] the integration over photon angles is performed in a coordinate system with $z$-axis in the direction of $p_{1}-p_{2}$, in which the photon angles are $\theta_{k}, \phi_{k}$. The momenturn transfer to the atom,

$$
q^{2}=\left(\underline{p}_{1}-\underline{p}_{2}-\underline{k}\right)^{2}=\left(\underline{p}_{1}-\underline{p}_{2}\right)^{2}+k^{2}-2 k\left|\underline{p}_{1}-\underline{p}_{2}\right| \cos \theta_{k}
$$

is independent of the azimuthal angle, $\phi_{k}$, of the photon. The integration over $\phi_{k}$ is then straightforward and leads to the expression given below in (2), while the integration over $\cos \theta_{k}$ may be written as an integration over $q^{2}$, as in (1).

Note that we have replaced $d k$ in [1] by $d \epsilon_{2}$. In the absence of recoil this makes no difference since then $k=\epsilon_{1}-\epsilon_{2}$ and hence $d k=-d \epsilon_{2}$. However, since in the present investigation the final electron is observed, we find it more reasonable to use $d \epsilon_{2}$. 


$$
\begin{aligned}
& \{\}=-\frac{2 k}{\left(2 \lambda+k^{2}\right)^{\frac{1}{2}}} \\
& -k\left(\frac{1}{D_{1}^{\frac{1}{2}}}-\frac{1}{D_{2}^{\frac{1}{2}}}\right)\left(\frac{q^{4}+4 \lambda^{2}-4 q^{2}\left(\epsilon_{1}^{2}+\epsilon_{2}^{2}-1\right)-16 \epsilon_{1} \epsilon_{2}}{2 \lambda-q^{2}}\right) \\
& +2 k \frac{\left(4 \epsilon_{1}^{2}-q^{2}\right)}{D_{1}^{3 / 2}}\left[2 \lambda\left(\lambda-k \epsilon_{2}\right)-\left(\lambda+k \epsilon_{1}\right) q^{2}\right] \\
& -2 k \frac{\left(4 \epsilon_{1}^{2}-q^{2}\right)}{D_{2}^{3 / 2}}\left[2 \lambda\left(\lambda+k \epsilon_{1}\right)-\left(\lambda-k \epsilon_{2}\right) q^{2}\right]
\end{aligned}
$$

with

$$
\begin{aligned}
& \lambda=\epsilon_{1} \epsilon_{2}-p_{1} p_{2} \cos \vartheta-1=\frac{1}{2}\left(\left|\underline{p}_{1}-\underline{p}_{2}\right|^{2}-k^{2}\right) \\
& \lambda_{0}=\epsilon_{1} \epsilon_{2}-p_{1} p_{2}-1 \\
& D_{1}=\left\{p_{1}\left(q^{2}-q_{2}^{2}\right)+2 p_{2} \lambda_{0} \cos \vartheta\right\}^{2}+4 k^{2} p_{2}{ }^{2} \sin ^{2} \vartheta \\
& D_{2}=\left\{p_{2}\left(q^{2}-q_{1}{ }^{2}\right)+2 p_{1} \lambda_{0} \cos \vartheta\right\}^{2}+4 k^{2} p_{1}{ }^{2} \sin ^{2} \vartheta \\
& q_{m}=\left|\underline{p}_{1}-\underline{p}_{2}\right|-k=\left(2 \lambda+k^{2}\right)^{\frac{1}{2}}-k \\
& q_{M}=\left|\underline{p}_{1}-\underline{p}_{2}\right|+k=\left(2 \lambda+k^{2}\right)^{\frac{1}{2}}+k \\
& q_{2}=2 p_{2} \sin \frac{1}{2} \vartheta \\
& q_{1}=2 p_{1} \sin \frac{1}{2} \vartheta \\
& \delta=p_{1}-p_{2}-k \\
& k=\epsilon_{1}-\epsilon_{2} .
\end{aligned}
$$


Here $d \delta=\sin \vartheta d \vartheta d \phi$ refers to the final electron, with polar and azimuthal angles $\vartheta, \phi$ in a coordinate system with $z$-axis in the direction of the initial electron.

$$
\begin{aligned}
& \epsilon_{1}, \underline{p}_{1} \text { are the energy and momentum of the incident electron. } \\
& \epsilon_{2}, \underline{p}_{2} \text { are the energy and momentum of the final electron. } \\
& k=\epsilon_{1}-\epsilon_{2} \text { is the energy of the emitted photon. }
\end{aligned}
$$

All of the energies and momenta are measured in the laboratory system, in units of $m c^{2}$ and $\mathrm{mc}$, respectively.

Having performed the integration over photon directions, giving (1) and (2), we are now confronted by a rather complicated looking expression which cannot be integrated analytically because of the presence of the screening function, which is in general only given numerically. However, if we consider the screening correction rather than the cross section itself, then a considerable simplification results: For the cross section with screening we write

$$
\begin{aligned}
d \sigma_{\text {scr }} & =\left(d \sigma_{\text {scr }}-d \sigma_{\text {unscr }}\right)+d \sigma_{\text {unscr }} \\
& \equiv \Delta \sigma_{\text {scr }}+d \sigma_{\text {unscr }} .
\end{aligned}
$$

In the integral in (1) we now write

$$
(1-F)^{2}=\left[(1-F)^{2}-1\right]+1 .
$$

The term $\left[(1-F)^{2}-1\right]$ gives the screening correction, $\Delta \sigma_{\text {scr }}$; the last term, 1, gives the unscreened cross section, $d \sigma_{\text {unscr }}$. This separation, which was used in [6] in integrating over the angles of the fina? 
electron, has two important consequences. First, the integration required to obtain the unscreened cross section can then be performed in closed form. In the present case, in which we integrate over photon angles, the unscreened cross section is given in [1] (p. B1346, eq (6)). Second, in the screening correction only very small values of $q$ are significant, viz.,

$$
q \lesssim O(\beta) \quad
$$

where $\beta$ is the inverse atomic screening radius. (In the Thomas-Fermi model, $\beta \approx \frac{Z^{1 / 3}}{121}<1$.) This may be seen by noting that we can write, approximately ([6], p. 897, eq $(6.30))$

$$
\frac{1-F}{q^{2}}=\frac{1}{q^{2}+\beta^{2}}
$$

and hence

$$
\frac{(1-F)^{2}-1}{q^{4}}=-\frac{2 q^{2} B^{2}+\beta^{4}}{\left(q^{2}+B^{2}\right)^{2} q^{4}} \text {. }
$$

Thus in the intearal for the screening correction, the contribution from $q \geq O(1)$ is of order $B^{2}$ and may be neglected. The only non-negligible contribution to the integral comes from $q \lesssim O(s)$ and, as we will show, a remarkable simplification of the integrand in (1) results in this region of very small momentum transfers. In $f$ act, we find that the screening correction to the angular distribution of the scattered electron (integrated over photon angles) is the same as the previously obtained [6] 
screening correction to the angular distribution of emitted photons (integrated over final electron angles), once one has made the appropriate change of variable from photon scattering angle to electron scattering angle.

The explicit expressions for the screening correction and the unscreened cross section (the angular distribution of scattered electrons, integrated over photon angles) derived in this report are given in the text by eqs (61), (66), and (67) (for the correction for intermediate screening), by eqs (61), (66), and (71) (for the correction for complete screening, and by eqs (63a) and (63b) for the unscreened cross section. 
We first make a few preliminary observations concerning the variables defined in (3) which are useful for the simplification of the integrand, (2), for the screening correction. In order to simplify the order of magnitude observations that follow, we will not only assume high energies of both the incident and final electron,

$$
\epsilon_{1} \gg 1 \quad, \quad \epsilon_{2} \gg 1
$$

but will assume as well that the photon energy is of the same order of magnitude as these energies:

$$
k=0(\epsilon) \text {. }
$$

A more detailed analysis is required to show that our final results are valid for somewhat smaller photon energies, but we will not present that here.

In the following analysis we show that, as a consequence of (9) and (10), the variables in (3) must satisfy certain restrictive conditions in order that we have a non-negligible screening correction. A very significant simplification of the integrand then follows as a result of these conditions, which are

$$
\begin{aligned}
& \text { i) } \epsilon>0(1 / \beta) \\
& \text { ii) } \vartheta^{2} \leqslant O(\beta / \epsilon) \leqslant O\left(\beta^{2}\right) \\
& \text { iii) } \quad D(1) \lesssim \lambda \lesssim O(\epsilon \beta) \text {. }
\end{aligned}
$$


From $i i i)$ it follows, then, since the significant values of $q$ in the integrand for the screening correction are $q \lesssim O(B)$, that we can set

$$
\frac{q^{2}}{\lambda} \lesssim 0\left(\beta^{2}\right) \ll 1
$$

in the integrand. Most importantly, this permits us to expand the denominators $\mathrm{O}_{1}{ }^{1 / 2}, \mathrm{O}_{2}{ }^{1 / 2}, \mathrm{O}_{1}{ }^{3 / 2}$, and $\mathrm{O}_{2}{ }^{3 / 2}$ in powers of $\frac{\mathrm{q}^{2}}{\lambda}$, as a result of which we find a very simple form for the integrand.

From (3), the minimum momentum transfer, $a_{m}$, is given by

$$
q_{m}=\left|\underline{p}_{1}-\underline{p}_{2}\right|-k \geqq p_{1}-p_{2}-k \equiv \delta
$$

and from (9) and (10) (see appendix)

$$
\delta \approx \frac{k}{2 \epsilon_{1} \epsilon_{2}}=0(1 / \epsilon) .
$$

Now if we are to have a non-negligible screening correction, we must have

$$
q_{m} \lesssim O(\beta)
$$

and hence also $\delta \leqslant O(\beta)$, from which we have restriction (i),

$$
\epsilon \geq O(1 / \beta)
$$

Next, from (3) we al so have 


$$
\begin{aligned}
a_{m} & =\left(2 \lambda+k^{2}\right)^{\frac{1}{2}}-k \\
& =\frac{2 \lambda}{\left(2 \lambda+k^{2}\right)^{\frac{1}{2}}+k} .
\end{aligned}
$$

Thus from (10) we have $\left(2 \lambda+k^{2}\right)^{\frac{2}{2}}+k=0(\epsilon)$ and $q_{m}=0(\lambda / \epsilon)$. Again in order that $q_{m} \leqslant 0(\beta)$ we now must require

$$
\lambda \lesssim O(\epsilon \beta)
$$

But at high energies we have (see appendix)

$$
\begin{aligned}
\lambda & \approx \frac{k^{2}}{2 \epsilon_{1} \epsilon_{2}}+2 \epsilon_{1} \epsilon_{2} \sin ^{2} \frac{3}{2} \vartheta \\
& =0(1)+O\left(\epsilon^{2} \vartheta^{2}\right) \gtrsim O(1) .
\end{aligned}
$$

Now from (15) and (16) we have

$$
\epsilon^{2} \vartheta^{2} \leq O(\epsilon 8)
$$

which, together with (11), gives ( $i i)$ :

$$
\imath^{2} \lesssim O(\beta / \epsilon) \leqslant O\left(\beta^{2}\right)
$$

Again from (15) and (16),

$$
0(1) \lesssim \lambda \leqslant 0(\epsilon \beta)
$$

which is ( $i i i)$. 
As a prelude to writing the explicit expression for the expansion of the integrand $\left(\{\}\right.$ in (2)) in powers of $q^{2 / \lambda}$, we examine the order of magnitude of each of the terms there. We show that, individually, the largest terms there are each of order $\frac{k^{2}}{\lambda^{2}} \geqslant O\left(1 / \beta^{2}\right) \gg 1$. However, taken together they cancel each other almost completely, and as a consequence we must keep the terms of order 1 in \{\} , these being of order $\beta^{2}$ relative to the individually large terms. To see this clearly we first write the expressions for $D_{1}$ and $D_{2}$ in a form more suitable for an expansion in powers of $q^{2} / \lambda$. (The expressions given in (3) are taken directly from [1].) From the appendix of the present report we have

$$
D_{1}=4 p_{2}^{2} \lambda^{2}-4\left[\left(\epsilon_{1} \epsilon_{2}-1\right) \lambda-k^{2}\right] q^{2}+p_{1}^{2} q^{4}
$$

and

$$
D_{2}=4 p_{1}^{2} \lambda^{2}-4\left[\left(\epsilon_{1} \epsilon_{2}-1\right) \lambda-k^{2}\right] q^{2}+p_{2}^{2} q^{4} .
$$

Now from (11) we note that in (19a) and (19b) the second term is of order

$$
\frac{q^{2}}{\lambda} \leq O\left(\beta^{2}\right)
$$

relative to the first term, and the third term is of order

$$
\left(q^{2} / \lambda\right)^{2} \lesssim O\left(\beta^{4}\right)
$$

relative to the first term. Thus we can write 


$$
\begin{aligned}
& D_{1}^{\frac{1}{2}}=2 p_{2} \lambda\left(1+O\left(\beta^{2}\right)\right) \\
& D_{2}^{\frac{1}{2}}=2 p_{1} \lambda\left(1+O\left(\beta^{2}\right)\right) .
\end{aligned}
$$

Again referring to (2), we note from (11) and (18) that

$$
\begin{gathered}
\left(\frac{q^{4}+4 \lambda^{2}-4 q^{2}\left(\epsilon_{1}{ }^{2}+\epsilon_{2}{ }^{2}-1\right)-16 \epsilon_{1} \epsilon_{2}}{2 \lambda-q^{2}}\right)=\frac{-16 \epsilon_{1} \epsilon_{2}}{2 \lambda}\left(1+O\left(\beta^{2}\right)\right) \\
{\left[2 \lambda\left(\lambda-k \epsilon_{2}\right)-\left(\lambda+k \epsilon_{1}\right) q^{2}\right]=2 \lambda\left(\lambda-k \epsilon_{2}\right)\left(1+o\left(\beta^{2}\right)\right)} \\
{\left[2 \lambda\left(\lambda+k \epsilon_{1}\right)-\left(\lambda-k \epsilon_{2}\right) q^{2}\right]=2 \lambda\left(\lambda+k \epsilon_{1}\right)\left(1+O\left(\beta^{2}\right)\right) .}
\end{gathered}
$$

Furthermore, in (22) and (23) we can write, from (3),

$$
\begin{aligned}
\lambda-k \epsilon_{2} & =\epsilon_{1} \epsilon_{2}-p_{1} p_{2} \cos \vartheta-1-\epsilon_{1} \epsilon_{2}+\epsilon_{2}^{2} \\
& =p_{2}^{2}-p_{1} p_{2} \cos \vartheta \\
& =-p_{2}\left(p_{1}-p_{2}\right)+2 p_{1} p_{2} \sin ^{2} \frac{1}{2} \vartheta
\end{aligned}
$$

and

$$
\begin{aligned}
\lambda+k \epsilon_{1} & =\epsilon_{1} \epsilon_{2}-p_{1} p_{2} \cos \vartheta-1+\epsilon_{1}^{2}-\epsilon_{1} \epsilon_{2} \\
& =p_{1}^{2}-p_{1} p_{2} \cos \vartheta \\
& =p_{1}\left(p_{1}-p_{2}\right)+2 p_{1} p_{2} \sin ^{2} \frac{1}{2} \vartheta .
\end{aligned}
$$


Now for high energies, $p_{1}-p_{2} \approx \epsilon_{1}-\epsilon_{2}=k$. Thus, assuming $k=0(\epsilon)$ and noting that $\vartheta^{2} \leqslant O(\beta / \epsilon)$ from (17), we have

$$
\begin{aligned}
& \lambda-k \epsilon_{2}=-p_{2}\left(p_{1}-p_{2}\right)(1+o(\beta / \epsilon)) \\
& \lambda+k \epsilon_{1}=p_{1}\left(p_{1}-p_{2}\right)(1+o(\beta / \epsilon)) .
\end{aligned}
$$

We now collect the terms in \{\} with a power of $D_{1}$ or $D_{2}$ in the denominator. From $(20 a),(20 b)$, and (21) we have, for the terms with $D_{1}^{-\frac{1}{2}}$ and $\mathrm{D}_{2}{ }^{-\frac{1}{2}}$ :

$-k\left(\frac{1}{D_{1}^{\frac{3}{2}}}-\frac{1}{D_{2}^{\frac{2}{2}}}\right)\left(\frac{q^{4}+4 \lambda^{2}-4 q^{2}\left(\epsilon_{1}^{2}+\epsilon_{2}^{2}-1\right)-16 \epsilon_{1} \epsilon_{2}}{2 \lambda-q^{2}}\right)=\frac{4 k \epsilon_{1} \epsilon_{2}}{2 \lambda^{2}}\left(\frac{1}{p_{2}}-\frac{1}{p_{1}}\right)\left(1+0\left(\beta^{2}\right)\right)$

$$
=\frac{4 k\left(p_{1}-p_{2}\right)}{\lambda^{2}} \frac{\epsilon_{1} \epsilon_{2}}{p_{1} p_{2}}\left(1+o\left(\beta^{2}\right)\right) \text {. }
$$

Next, substituting (26) and (27) in (22) and (23) and using (20a) and (20b), we have, for the terms with $D_{1}^{-3 / 2}$ and $D_{2}^{-3 / 2}$ :

$$
\frac{2 k\left(4 \epsilon_{2}{ }^{2}-q^{2}\right)}{D_{1}{ }^{3 / 2}}\left[2 \lambda\left(\lambda-k \epsilon_{2}\right)-\left(\lambda+k \epsilon_{1}\right) q^{2}\right]=\frac{-2 k\left(p_{1}-p_{2}\right)}{\lambda^{2}} \frac{\epsilon_{2}{ }^{2}}{p_{2}{ }^{2}}\left(1+O\left(\beta^{2}\right)\right)
$$

and

$$
\frac{-2 k\left(4 \epsilon_{1}^{2}-q^{2}\right)}{D_{2}{ }^{3 / 2}}\left[2 \lambda\left(\lambda+k \epsilon_{1}\right)-\left(\lambda-k \epsilon_{2}\right) q^{2}\right]=\frac{-2 k\left(p_{1}-p_{2}\right)}{\lambda^{2}} \frac{\epsilon_{1}^{2}}{p_{1}^{2}}\left(1+O\left(\beta^{2}\right)\right) .
$$


Thus the large terms in \{\} , those exhibited specifically in (28), (29), and (30), are indeed each of order $\left.\frac{k^{2}}{\lambda^{2}} \geq 01 / \beta^{2}\right) \gg 1$, as mentioned. However, when added they give

$$
\frac{2 k\left(p_{1}-p_{2}\right)}{\lambda^{2}}\left(\frac{\epsilon_{1}}{p_{1}}-\frac{\epsilon_{2}}{p_{2}}\right)^{2}=0\left(\frac{k^{2}}{\lambda^{2}} \cdot \frac{k^{2}}{\epsilon^{6}}\right)
$$

since, as shown in the appendix,

$$
\left(\frac{\epsilon_{1}}{p_{1}}-\frac{\epsilon_{2}}{p_{2}}\right)^{2}=0\left(\frac{k^{2}}{\epsilon^{6}}\right)
$$

Thus the "large" terms cancel, leaving a remainder of order

$$
\frac{k^{4}}{\lambda^{2} \epsilon^{6}}=\left(\frac{k^{2}}{\lambda \epsilon^{2}}\right)^{2} \frac{1}{\epsilon^{2}} \lesssim \frac{1}{\epsilon^{2}}
$$

since from (16) $\frac{\lambda \epsilon^{2}}{k^{2}} \gtrsim O(1)$. This remainder can therefore be neglected; the contribution to be retained thus comes from the terms of $0(1)$, i.e., from the terms indicated in $(20 a)-(23)$ as being of $O\left(\beta^{2}\right)$ relative to the individually large terms. It should be noted that the very significant cancellation, giving the factor

$$
\left(\frac{\epsilon_{1}}{p_{1}}-\frac{\epsilon_{2}}{p_{2}}\right)^{2}=0\left(\frac{k^{2}}{\epsilon^{6}}\right) \lesssim 0\left(1 / \epsilon^{4}\right)
$$


results from our particular choice for the "large" terms in (28)-(30). Had we made a less auspicious choice, for example by writing

$$
\begin{aligned}
& \lambda-k \epsilon_{2}=-k \epsilon_{2}(1+0(\beta / \epsilon)) \\
& \lambda+k \epsilon_{1}=k \epsilon_{1}(1+0(\beta / \epsilon))
\end{aligned}
$$

instead of (26) and (27), then the large terms would only have cancelled to relative order $1 / \epsilon^{2}$ (rather than to relative order $1 / \epsilon^{4}$ ). The remainder would then have been of order 1, and have had to be retained. 
IV. DERIVATION OF THE SCREENING CORRECTION

We now proceed with the expansion of the terms in \{\} in (2), retaining all terms of $0(1)$, i.e., of order $\beta^{2}$ relative to the large terms which have just been shown to cancel. However, we neglect terms which are individually of $O\left(\beta^{2}\right)$ or of $0\left(1 / \epsilon^{2}\right)$; these terms are of order $\beta^{4}$ or of order $\beta^{2} / \epsilon^{2}$ relative to the large terms.

Referring to (2), we have first

$$
\begin{aligned}
\frac{-2 k}{\left(2 \lambda+k^{2}\right)^{\frac{1}{2}}} & =\frac{-2}{\left(1+\frac{2 \lambda}{k^{2}}\right)^{\frac{1}{2}}} \\
& =-2+0\left(\lambda / k^{2}\right) .
\end{aligned}
$$

Here, from (18), (10), and (13),

$$
\frac{\lambda}{k^{2}} \lesssim O(\beta / \epsilon) \lesssim O\left(\beta^{2}\right)
$$

Thus, as just explained, we retain the term -2, but neglect the term of $O\left(\lambda / k^{2}\right) \lesssim O\left(\beta^{2}\right)$, writing

$$
\frac{-2 k}{\left(2 \lambda+k^{2}\right)^{\frac{2}{2}}}=-2\left(1+o\left(\beta^{2}\right)\right) \text {. }
$$

Next, in the term with factors $D_{1}^{-\frac{1}{2}}$ and $D_{2}^{-\frac{1}{2}}$ we expand the factors 
$\left(\frac{q^{4}+4 \lambda^{2}-4 q^{2}\left(\epsilon_{1}^{2}+\epsilon_{2}^{2}-1\right)-16 \epsilon_{1} \epsilon_{2}}{2 \lambda-q^{2}}\right)$

$$
=\left[-\frac{8 \epsilon_{1} \epsilon_{2}}{\lambda}-\frac{2 q^{2}\left(\epsilon_{1}{ }^{2}+\epsilon_{2}{ }^{2}\right)}{\lambda}+2 \lambda-\frac{4 \epsilon_{1} \epsilon_{2} q^{2}}{\lambda^{2}}\right]\left(1+0\left(\beta^{4}\right)\right)
$$

and, from (19a) and (19b),

$$
\begin{aligned}
-k\left(\frac{1}{D_{1}^{\frac{1}{2}}}-\frac{1}{D_{2}^{\frac{1}{2}}}\right)= & -\frac{k}{2 p_{2} \lambda}\left[1+\frac{1}{2} \frac{\left(\epsilon_{1} \epsilon_{2} \lambda-k^{2}\right) q^{2}}{p_{2}{ }^{2} \lambda^{2}}\right]\left(1+0\left(\beta^{4}\right)\right) \\
& +\frac{k}{2 p_{1} \lambda}\left[1+\frac{1}{2} \frac{\left(\epsilon_{1} \epsilon_{2} \lambda-k^{2}\right) q^{2}}{p_{1}{ }^{2} \lambda^{2}}\right]\left(1+0\left(\beta^{4}\right)\right) \\
= & -\frac{k}{2 \lambda} \frac{\left(p_{1}-p_{2}\right)}{p_{1} p_{2}}\left[1+\frac{k\left(p_{1}{ }^{2}+p_{1} p_{2}+p_{2}{ }^{2}\right) q^{2}}{2 p_{1}{ }^{2} p_{2}{ }^{2} \lambda^{2}}\right]\left(1+O\left(\beta^{4}\right)\right) .
\end{aligned}
$$

Multiplying the factors in (36) and (37) then gives the exparsion of the term in (2) with factors $\mathrm{O}_{1}^{-\frac{1}{2}}$ and $\mathrm{O}_{2}^{-\frac{1}{2}}$ : 


$$
\begin{aligned}
-k\left(\frac{1}{D_{1}^{\frac{3}{2}}}-\frac{1}{D_{2}{ }^{\frac{1}{2}}}\right)\left(\frac{q^{4}+4 \lambda^{2}-4 q^{2}\left(\epsilon_{1}{ }^{2}+\epsilon_{2}{ }^{2}-1\right)-16 \epsilon_{1} \epsilon_{2}}{2 \lambda-q^{2}}\right) \\
=-\frac{k\left(p_{1}-p_{2}\right)}{2 \lambda p_{1} p_{2}}\left[1+\frac{\left(\epsilon_{1} \epsilon_{2} \lambda-k^{2}\right)\left(p_{1}{ }^{2}+p_{1} p_{2}+p_{2}{ }^{2}\right) q^{2}}{2 p_{1}{ }^{2} p_{2}{ }^{2} \lambda^{2}}\right] \\
\times\left[-\frac{8 \epsilon_{1} \epsilon_{2}}{\lambda}-\frac{2 q^{2}\left(\epsilon_{1}{ }^{2}+\epsilon_{2}{ }^{2}\right)}{\lambda}+2 \lambda-\frac{4 \epsilon_{1} \epsilon_{2} q^{2}}{\lambda^{2}}\right]\left(1+0\left(\beta^{4}\right)\right) \\
=\frac{4 k\left(p_{1}-p_{2}\right)}{2 \lambda} \frac{\epsilon_{1} \epsilon_{2}}{p_{1} p_{2}} \\
\\
\quad-\frac{k\left(p_{1}-p_{2}\right)}{p_{1} p_{2}} \\
+\frac{k\left(p_{1}-p_{2}\right) q^{2}}{p_{1} p_{2} \lambda}\left[\frac{2 \epsilon_{1} \epsilon_{2}\left(\epsilon_{1} \epsilon_{2} \lambda-k^{2}\right)\left(p_{1}{ }^{2}+p_{1} p_{2}+p_{2}{ }^{2}\right)}{p_{1}{ }^{2} p_{2}{ }^{2} \lambda^{3}}+\frac{\left(\epsilon_{1}{ }^{2}+\epsilon_{2}{ }^{2}\right)}{\lambda}+\frac{2 \epsilon_{1} \epsilon_{2}}{\lambda^{2}}\right]
\end{aligned}
$$$$
+ \text { terms of relative order } \beta^{4} \text {. }
$$ 
Finally, we have the terms in (2) with factors $D_{1}^{-3 / 2}$ and $D_{2}^{-3 / 2}$. Again we expand, substituting (24) and (25) in the factors in these terms:

$$
\begin{aligned}
2 \lambda\left(\lambda-k \epsilon_{2}\right)-\left(\lambda+k \epsilon_{1}\right) q^{2}= & 2 \lambda\left[-p_{2}\left(p_{1}-p_{2}\right)+2 p_{1} p_{2} \sin ^{2 \frac{3}{2} \vartheta}\right] \\
& -\left[p_{1}\left(p_{1}-p_{2}\right)+2 p_{1} p_{2} \sin ^{2 \frac{3}{2} \vartheta}\right] q^{2} \\
= & {\left[-2 \lambda p_{2}\left(p_{1}-p_{2}\right)+4 \lambda p_{1} p_{2} \sin ^{2 \frac{1}{2} \vartheta}\right.} \\
& \left.-p_{1}\left(p_{1}-p_{2}\right) q^{2}\right]\left(1+o\left(\beta^{2} / \epsilon^{2}\right)\right)
\end{aligned}
$$

$2 \lambda\left(\lambda+k \epsilon_{1}\right)-\left(\lambda-k \epsilon_{2}\right) q^{2}=2 \lambda\left[p_{1}\left(p_{1}-p_{2}\right)+2 p_{1} p_{2} \sin ^{2 \frac{1}{2} \vartheta}\right]$

$$
\begin{gathered}
-\left[-p_{2}\left(p_{1}-p_{2}\right)+2 p_{1} p_{2} \sin ^{2 \frac{1}{2} \vartheta}\right] q^{2} \\
=\left[2 \lambda p_{1}\left(p_{1}-p_{2}\right)+4 \lambda p_{1} p_{2} \sin ^{2 \frac{1}{2} \vartheta}\right. \\
\left.+p_{2}\left(p_{1}-p_{2}\right) q^{2}\right]\left(1+0\left(\beta^{2} / \epsilon^{2}\right)\right) .
\end{gathered}
$$

We then expand $D_{1}^{-3 / 2}$ and $D_{2}^{-3 / 2}$, using (19a) and (19b), from which

$$
\begin{aligned}
& D_{1}^{-3 / 2}=\frac{1}{\left(2 p_{2} \lambda\right)^{3}}\left[1+\frac{3}{2} \frac{\left(\epsilon_{1} \epsilon_{2} \lambda-k^{2}\right) q^{2}}{p_{2}{ }^{2} \lambda^{2}}\right]\left(1+0\left(\beta^{4}\right)\right) \\
& D_{2}^{-3 / 2}=\frac{1}{\left(2 p_{1} \lambda\right)^{3}}\left[1+\frac{3}{2} \frac{\left(\epsilon_{1} \epsilon_{2} \lambda-k^{2}\right) q^{2}}{p_{1}{ }^{2} \lambda^{2}}\right]\left(1+0\left(\beta^{4}\right)\right)
\end{aligned}
$$


Then, referring to the terms in (2), we have, from (39a) and (40),

$$
\begin{aligned}
2 k \frac{\left(4 \epsilon_{2}{ }^{2}-q^{2}\right)}{D_{1}{ }^{3 / 2}}[ & \left.2 \lambda\left(\lambda-k \epsilon_{2}\right)-\left(\lambda+k \epsilon_{1}\right) q^{2}\right] \\
& =\frac{k \epsilon_{2}{ }^{2}}{p_{2}{ }^{3} \lambda^{3}}\left[1+\frac{3}{2} \frac{\left(\epsilon_{1} \epsilon_{2} \lambda-k^{2}\right)}{p_{2}{ }^{2} \lambda^{2}} q^{2}\right] \\
& \times\left[-2 \lambda p_{2}\left(p_{1}-p_{2}\right)+4 \lambda p_{1} p_{2} \sin ^{2 \frac{1}{2} \vartheta}-p_{1}\left(p_{1}-p_{2}\right) q^{2}\right]\left(1+0\left(\beta^{4}\right)\right) \\
= & -\frac{2 k\left(p_{1}-p_{2}\right)}{\lambda^{2}} \frac{\epsilon_{2}{ }^{2}}{p_{2}{ }^{2}} \\
& +\frac{4 k p_{1}}{\lambda^{2}} \frac{\epsilon_{2}{ }^{2}}{p_{2}{ }^{2}} \sin ^{2 \frac{1}{2} \vartheta} \\
& -\frac{k\left(p_{1}-p_{2}\right)}{\lambda^{4}} \frac{\epsilon_{2}{ }^{2}}{p_{2}{ }^{4}} q^{2}\left[3\left(\epsilon_{1} \epsilon_{2} \lambda-k^{2}\right)+p_{1} p_{2} \lambda\right]
\end{aligned}
$$

+ terms of relative order $\beta^{4}$. 
Finally, from (39b) and (41) we have

$-2 k \frac{\left(4 \epsilon_{1}^{2}-q^{2}\right)}{0_{2}^{3 / 2}}\left[2 \lambda\left(\lambda+k \epsilon_{1}\right)-\left(\lambda-k \epsilon_{2}\right) q^{2}\right]$

$$
\begin{aligned}
& =-\frac{k \epsilon_{1}{ }^{2}}{p_{1}{ }^{3} \lambda^{3}}\left[1+\frac{3}{2} \frac{\left(\epsilon_{1} \epsilon_{2} \lambda-k^{2}\right)}{p_{1}{ }^{2} \lambda^{2}} q^{2}\right] \\
& \times\left[2 \lambda p_{1}\left(p_{1}-p_{2}\right)+4 \lambda p_{1} p_{2} \sin 2 \frac{3}{2} \vartheta+p_{2}\left(p_{1}-p_{2}\right) q^{2}\right]\left(1+0\left(\beta^{4}\right)\right) \\
& =-\frac{2 k\left(p_{1}-p_{2}\right)}{\lambda^{2}} \frac{\epsilon_{1}^{2}}{p_{1}^{2}} \\
& -\frac{4 k p_{2}}{\lambda^{2}} \frac{\epsilon_{1}^{2}}{p_{1}^{2}} \sin ^{2} \frac{z_{2} \vartheta}{2} \\
& -\frac{k\left(p_{1}-p_{2}\right)}{\lambda^{4}} \frac{\epsilon_{1}{ }^{2}}{p_{1}^{4}} q^{2}\left[3\left(\epsilon_{1} \epsilon_{2} \lambda-k^{2}\right)+p_{1} p_{2} \lambda\right] \\
& + \text { terms of relative order } B^{4} \text {. }
\end{aligned}
$$

Now the first term on the right hand side of each of the three expressions - (38), (42), and (43) - are the large terms we have already considered (note eqs. (28), (29), and (30)). As mentioned, they are individually of order $k^{2} / \lambda^{2}=O\left(\epsilon^{2}\right)$, but when added give a contribution of $O\left(1 / \epsilon^{2}\right)$, which we neglect (see eqs (31) and (32)). We are thus left with the remaining terms, of $0(1)$, in $(38),(42)$, and (43), as well as the term -2 from eqs $(33)-(35)$. We first gather the terms without a factor $q^{2}$. These come from (35) and the second term on the right hand side of eqs (38), (42), and (43). They are 


$$
-2-\frac{k\left(p_{1}-p_{2}\right)}{p_{1} p_{2}}+\frac{4 k p_{1}}{\lambda^{2}} \frac{\epsilon_{2}^{2}}{p_{2}{ }^{2}} \sin ^{2} \frac{1}{2} \vartheta-\frac{4 k p_{2}}{\lambda^{2}} \frac{\epsilon_{1}{ }^{2}}{p_{1}{ }^{2}} \sin ^{2} \frac{1}{2} \vartheta .
$$

Since these terms are each of $O(1)$, we may now set $\epsilon_{1} \approx p_{1}, \epsilon_{2} \approx p_{2}$ throughout, neglecting terms of $0\left(1 / \epsilon^{2}\right)$. We then have

$$
-2-\frac{k^{2}}{\epsilon_{1} \epsilon_{2}}+\frac{4 k^{2}}{\lambda^{2}} \sin ^{2} \frac{1}{2} \vartheta=-\frac{\left(\epsilon_{1}{ }^{2}+\epsilon_{2}{ }^{2}\right)}{\epsilon_{2} \epsilon_{2}}+\frac{4 k^{2}}{\lambda^{2}} \sin ^{2} \frac{1}{2} \vartheta .
$$

We next gather the terms with a factor $q^{2}$. These come from the last term on the right hand side of eqs (38), (42), and (43). They are

$$
\begin{aligned}
& \frac{k\left(p_{1}-p_{2}\right)}{\lambda p_{1} p_{2}} q^{2}\left[\frac{2 \epsilon_{1} \epsilon_{2}\left(\epsilon_{1} \epsilon_{2} \lambda-k^{2}\right)\left(p_{1}{ }^{2}+p_{1} p_{2}+p_{2}{ }^{2}\right)}{p_{1}{ }^{2} p_{2}{ }^{2} \lambda^{3}}+\frac{\left(\epsilon_{1}{ }^{2}+\epsilon_{2}{ }^{2}\right)}{\lambda}+\frac{2 \epsilon_{1} \epsilon_{2}}{\lambda^{2}}\right] \\
& -\frac{k\left(p_{1}-p_{2}\right)}{\lambda^{4}} q^{2} \frac{\epsilon_{2}{ }^{2}}{p_{2}{ }^{4}}\left[3\left(\epsilon_{1} \epsilon_{2} \lambda-k^{2}\right)+p_{1} p_{2} \lambda\right] \\
& -\frac{k\left(p_{1}-p_{2}\right)}{\lambda^{4}} q^{2} \frac{\epsilon_{1}{ }^{2}}{p_{1}}\left[3\left(\epsilon_{1} \epsilon_{2} \lambda-k^{2}\right)+p_{1} p_{2} \lambda\right] .
\end{aligned}
$$

As with the terms without a factor $q^{2}$, listed in (44), we may now set $\epsilon_{1} \approx p_{1}, \epsilon_{2} \approx p_{2}$ throughout, again neglecting terms of $0\left(1 / \epsilon^{2}\right)$. The terms in (46) then become 


$$
\begin{aligned}
& \frac{k^{2} q^{2}}{\lambda^{2}}\left[\begin{array}{l}
\frac{2\left(\epsilon_{1} \epsilon_{2} \lambda-k^{2}\right)\left(\epsilon_{1}{ }^{2}+\epsilon_{1} \epsilon_{2}+\epsilon_{2}{ }^{2}\right)}{\epsilon_{1}{ }^{2} \epsilon_{2}{ }^{2} \lambda^{2}}+\frac{\left(\epsilon_{1}^{2}+\epsilon^{2}\right)}{\epsilon_{1} \epsilon_{2}}+\frac{2}{\lambda} \\
-\frac{3\left(\epsilon_{1} \epsilon_{2} \lambda-k^{2}\right)}{\epsilon_{2}{ }^{2} \lambda^{2}}-\frac{3\left(\epsilon_{1} \epsilon_{2} \lambda-k^{2}\right)}{\epsilon_{1}{ }^{2} \lambda^{2}}-\frac{1}{\lambda}\left(\frac{\epsilon_{1}}{\epsilon_{2}}+\frac{\epsilon_{2}}{\epsilon_{1}}\right)
\end{array}\right] \\
& =\frac{k^{2} q^{2}}{\lambda^{2}}\left[\begin{array}{l}
\frac{\left(\epsilon_{1} \epsilon_{2} \lambda-k^{2}\right)}{\lambda^{2}}\left[\frac{2}{\epsilon_{2}{ }^{2}}+\frac{2}{\epsilon_{1}{ }^{2}}+\frac{2}{\epsilon_{1} \epsilon_{2}}-\frac{3}{\epsilon_{1}{ }^{2}}-\frac{3}{\epsilon_{2}{ }^{2}}\right] \\
+\frac{2}{\lambda}+\frac{\left(\epsilon_{1}{ }^{2}+\epsilon_{2}{ }^{2}\right)}{\epsilon_{1} \epsilon_{2}}\left(1-\frac{1}{\lambda}\right)
\end{array}\right] \\
& =\frac{k^{2} q^{2}}{\lambda^{2}}\left[\frac{\left(\epsilon_{1} \epsilon_{2} \lambda-k k^{2}\right)}{\lambda^{2}}\left(1-\frac{1}{\epsilon_{1}{ }^{2}}-\frac{1}{\epsilon_{2}{ }^{2}}+\frac{2}{\epsilon_{1} \epsilon_{2}}\right)-\frac{1}{\epsilon_{1} \epsilon_{2} \lambda}\left(\epsilon_{1}^{2}+\epsilon_{2}{ }^{2}-2 \epsilon_{1} \epsilon_{2}\right)+\frac{\epsilon_{1}{ }^{2}+\epsilon_{2}{ }^{2}}{\epsilon_{1} \epsilon_{2}}\right] \\
& =\frac{k^{2} q^{2}}{\lambda^{2}}\left[-\frac{\left(\epsilon_{1} \epsilon_{2} \lambda-k^{2}\right) k^{2}}{\epsilon_{1}^{2} \epsilon_{2}^{2} \lambda^{2}}-\frac{k^{2}}{\epsilon_{1} \epsilon_{2} \lambda}+\frac{\left(\epsilon_{1}^{2}+\epsilon_{2}^{2}\right)}{\epsilon_{1} \epsilon_{2}}\right] \\
& =\frac{k^{2} q^{2}}{\lambda^{2}}\left[-\frac{2 k^{2}}{\epsilon_{1} \epsilon_{2} \lambda}+\frac{k^{4}}{\epsilon_{1}^{2} \epsilon_{2}^{2} \lambda^{2}}+\frac{\left(\epsilon_{1}^{2}+\epsilon_{2}^{2}\right)}{\epsilon_{1} \epsilon_{2}}\right] \\
& =\frac{k^{2} q^{2}}{\lambda^{2}}\left[\frac{\left(\epsilon_{1}^{2}+\epsilon_{2}^{2}\right)}{\epsilon_{1} \epsilon_{2}}-\frac{k^{2}}{\lambda^{2} \epsilon_{1} \epsilon_{2}}\left(2 \lambda-\frac{k^{2}}{\epsilon_{1} \epsilon_{2}}\right)\right] \text {. }
\end{aligned}
$$


Now for high energies (see (A4))

$$
2 \lambda \approx \frac{k^{2}}{\epsilon_{1} \epsilon_{2}}+4 \epsilon_{1} \epsilon_{2} \sin 2 \frac{1}{2} \vartheta
$$

and thus we have above, in (46),

$$
\frac{k^{2} q^{2}}{\lambda^{2}}\left[\frac{\left(\epsilon_{1}^{2}+\epsilon_{2}^{2}\right)}{\epsilon_{1} \epsilon_{2}}-\frac{4 k^{2}}{\lambda^{2}} \sin 2 \frac{1}{2} \vartheta\right] \text {. }
$$

We note that the terms in the factor [ ] here are identical to the terms on the right hand side of (45), apart from a sign. Thus, adding (45) and (47), we have, for the terms in \{\} in (2), the remarkably simple expression

$$
\{\}=\frac{k^{2}}{\lambda^{2} \epsilon_{1} \epsilon_{2}}\left[\epsilon_{1}{ }^{2}+\epsilon_{2}{ }^{2}-\frac{4 k^{2} \epsilon_{1} \epsilon_{2}}{\lambda^{2}} \sin 2 \frac{3}{2} \vartheta\right]\left(q^{2}-\frac{\lambda^{2}}{k^{2}}\right) .
$$

We now write this in a form which is similar to that obtained for the screening correction considered in [6], where we integrated over final electron directions. For high energies we have, from (A4),

$$
4 \epsilon_{1} \epsilon_{2} \sin 2 \frac{1}{2} \vartheta \approx 2 \lambda-\frac{k^{2}}{\epsilon_{1} \epsilon_{2}}
$$

and thus, in (48), 


$$
\begin{aligned}
\frac{4 k^{2} \epsilon_{1} \epsilon_{2} \sin ^{2} \frac{1}{2} \vartheta}{\lambda^{2}} & \approx \frac{k^{2}}{\lambda^{2}}\left(2 \lambda-\frac{k^{2}}{\epsilon_{1} \epsilon_{2}}\right) \\
& =\frac{2 k^{2}}{\lambda}\left(1-\frac{k^{2}}{2 \epsilon_{1} \epsilon_{2} \lambda}\right) \\
& \approx 4 \epsilon_{1} \epsilon_{2} \frac{\delta k}{\lambda}\left(1-\frac{\delta k}{\lambda}\right)
\end{aligned}
$$

on substituting $\delta \approx \frac{k}{2 \epsilon_{1} \epsilon_{2}}$ from $(A 1)$. The expression (48) for \{\} can thus be written in the form

$$
\{\}=\frac{k^{2}}{\lambda^{2} \epsilon_{1} \epsilon_{2}}\left[\epsilon_{1}{ }^{2}+\epsilon_{2}{ }^{2}-4 \epsilon_{1} \epsilon_{2} \frac{\delta k}{\lambda}\left(1-\frac{\delta k}{\lambda}\right)\right]\left(q^{2}-\frac{\lambda^{2}}{k^{2}}\right) \text {. }
$$

Now from (A7) and (A19) we have, for high energies and small scatiering angles,

$$
\frac{\lambda}{k} \approx \delta\left(1+w^{2}\right) \equiv \frac{\delta}{\chi}
$$

where $w$ is defined by (A6):

$$
w=\frac{\epsilon_{1} \epsilon_{2} \vartheta}{k}
$$

Substituting (50) in (49) then gives

$$
\{\}=\frac{\chi^{2}}{\delta^{2} \epsilon_{1} \epsilon_{2}}\left[\epsilon_{1}^{2}+\epsilon_{2}^{2}-4 \epsilon_{1} \epsilon_{2} \chi(1-\chi)\right]\left(q^{2}-\frac{\delta^{2}}{\chi^{2}}\right) .
$$


Finally, from the definition for $x,(A 19)$,

$$
x=\frac{1}{1+w^{2}}
$$

we have

$$
\chi(1-\chi)=w^{2} \chi^{2},
$$

which, when substituted in (52), gives

$$
\{\}=\frac{\chi^{2}}{\delta^{2} \epsilon_{1} \epsilon_{2}}\left[\epsilon_{1}{ }^{2}+\epsilon_{2}{ }^{2}-4 \epsilon_{1} \epsilon_{2} w^{2} \chi^{2}\right]\left(q^{2}-\frac{\delta^{2}}{\chi^{2}}\right) \text {. }
$$

In order to obtain the screening correction, we must substitute (53) in (1), and replace $(1-F)^{2}$ in the integrand by $(1-F)^{2}-1$, as indicated in (4) and (5). Further, in the lower limit in (1) we can write, from $(A 20)$,

$$
q_{m} \approx \frac{\delta}{\chi}
$$

and take the upper limit to be infinite since there is negligible contribution to the screeing correction from $q \geq 0(1)$. We then have

$$
\begin{aligned}
\frac{d \sigma_{s c r} \operatorname{cor} r=}{d \Omega} & \frac{1}{2 \pi} \frac{e^{2}}{\hbar c}\left(\frac{Z e^{2}}{m c^{2}}\right)^{2} \frac{d \epsilon_{2}}{\epsilon_{1}{ }^{2} k} \frac{\chi^{2}}{\delta^{2}}\left[\epsilon_{1}{ }^{2}+\epsilon_{2}{ }^{2}-4 \epsilon_{1} \epsilon_{2} w^{2} \chi^{2}\right] \\
& \times \int_{\delta^{2} / \chi^{2}}^{\infty} \frac{\left[(1-F)^{2}-1\right]}{q^{4}}\left(q^{2}-\frac{\delta^{2}}{\chi^{2}}\right) d\left(q^{2}\right)
\end{aligned}
$$


In order to compare this expression with the screening correction given in [6] we note from (50) and (51) that for small scattering angles, 9,

$$
\begin{aligned}
d \Omega & =\sin \vartheta d \vartheta d \phi \\
& \approx \vartheta d \vartheta d \phi \\
& =\frac{1}{2}\left(\frac{k}{\epsilon_{1} \epsilon_{2}}\right)^{2} d\left(w^{2}\right) d \phi \\
& =2 \delta^{2} d\left(w^{2}\right) d \phi \\
& =-2 \frac{\delta^{2}}{\chi^{2}} d \chi d \phi .
\end{aligned}
$$

Then, since the expression (55) is independent of $\phi$, integration over this variable merely gives a factor $2 \pi$. We then have, if we take $q$ rather than $q^{2}$ as the variable of integration,

$$
\begin{aligned}
d \sigma_{\text {scr corr }} & =4 \frac{e^{2}}{\hbar c}\left(\frac{Z e^{2}}{m c^{2}}\right)^{2} \frac{d \epsilon_{2}}{\epsilon_{1}{ }^{2} k} d \chi\left[\epsilon_{1}{ }^{2}+\epsilon_{2}{ }^{2}-4 \epsilon_{1} \epsilon_{2} w^{2} \chi^{2}\right] \\
& \times \int_{\delta / \chi}^{\infty} \frac{\left[(1-F)^{2}-1\right]}{q^{3}}\left(q^{2}-\frac{\delta^{2}}{\chi^{2}}\right) d q .
\end{aligned}
$$

Comparison with the cross section integrated over final electron directions, i.e., the angular distribution of the photons, given in [6], p. 897 , eq $(7.2)$, 
then shows that (57) is identical to the screening correction ${ }^{*}$ in [6], once one has made the substitutions

$$
\begin{gathered}
d k \rightarrow d \epsilon_{2} \\
u \rightarrow w \\
\xi=\frac{1}{1+u^{2}} \rightarrow \frac{1}{1+w^{2}}=x .
\end{gathered}
$$

Having already noted that in the absence of recoil $d k=-d \epsilon_{2}$, the significant replacement in (58) is

$$
u \rightarrow w
$$

where

$$
u=p_{1} \theta_{1} \quad \text { with } \quad \theta_{1}=\Varangle\left(\underline{p}_{1}, \underline{k}\right)
$$

is the convenient variable when the photon is observed, and

$$
w=\frac{p_{1} p_{2} \vartheta}{k} \quad \text { with } \quad \vartheta=\Varangle\left(\underline{p}_{1}, \underline{p}_{2}\right)
$$

\footnotetext{
* Note that the screening correction in [6] is contained in the terms in (7.2) with factor $\Gamma$, defined in $(6.29)$ and $(6.28)$ in that reference. It comes, specifically, from the term $\mathscr{I}(\delta / \xi)$ in $\Gamma$, as can be seen from eq $(6.28)$ there.
} 
is the corresponding variable when the final electron is observed. Indeed, a comparison of the two situations in the figure below

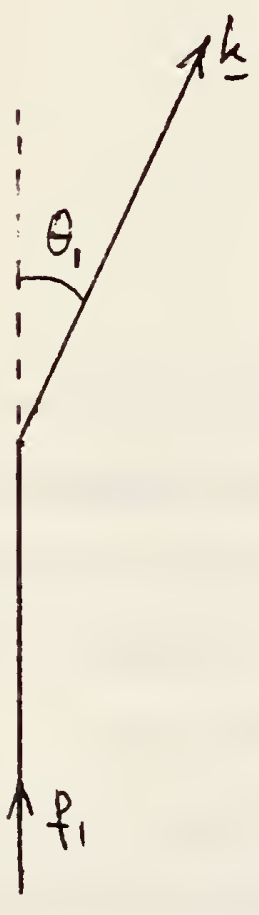

photon observed

integrate over electron angles

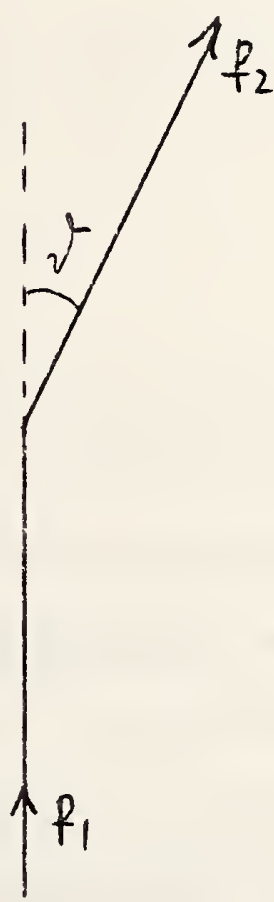

electron observed integrate over photon angles

suggests that the analogous variables might be

$$
k \theta_{1}
$$

(photon observed)

and

$$
p_{2} \vartheta
$$$$
\text { (electron observed) . }
$$

This would imply the substitution given in (58), since from

$$
k \theta_{1} \rightarrow p_{2} \vartheta
$$




$$
u=p_{1} \theta_{1}=\frac{p_{1}}{k}\left(k \theta_{1}\right) \rightarrow \frac{p_{1}}{k}\left(p_{2} \vartheta\right) \approx \frac{\epsilon_{1} \epsilon_{2}^{\vartheta}}{k}=w
$$

and

$$
\xi=\frac{1}{1+u^{2}} \rightarrow \frac{1}{1+w^{2}}=\chi .
$$

Having shown the identity of the screening correction derived here, eq (57), with that given earlier in [6] when integrating over electron angles, under the substitution (58), we choose finally to express it in terms of the variable $a_{m}$. It then has the same form whether one integrates over photon or electron angles, the only difference being the definition of the minimum momentum transfer, $a_{m}$, in terms of the remaining variables, viz.,

$$
\begin{array}{lc}
q_{m}=\left|\underline{p}_{1}-\underline{k}\right|-p_{2} & \text { photon observed } \\
q_{m}=\left|\underline{p}_{1}-\underline{p}_{2}\right|-k & \text { final electron observed }
\end{array}
$$

Substituting $q_{m}$ as given in (54) into the expression (55) we have 


$$
\begin{aligned}
\frac{d \sigma_{s c r} \operatorname{cor} r}{d \Omega} & =\frac{1}{2 \pi} \frac{e^{2}}{h c}\left(\frac{Z e^{2}}{m c^{2}}\right)^{2} \frac{d \epsilon_{2}}{\epsilon_{1}{ }^{2 k}}\left[\epsilon_{1}{ }^{2}+\epsilon_{2}{ }^{2}-4 \epsilon_{1} \epsilon_{2} \frac{\delta}{q_{m}}\left(1-\frac{\delta}{q_{m}}\right)\right] \\
& \times \int_{q_{m}{ }^{2}}^{\infty} \frac{\left[(1-F(q))^{2}-1\right]}{q^{4}}\left(\frac{q^{2}}{q_{m}{ }^{2}}-1\right) d\left(q^{2}\right)
\end{aligned}
$$

From (4) and (61) we now have our complete expression for the cross section integrated over photon angles:

$$
\frac{d \sigma}{d \Omega} \mathrm{scr}=\frac{d \sigma_{u n s c r}}{d \Omega}+\frac{d \sigma_{\text {scr }}}{d \Omega} \text { corr } .
$$

As noted earlier, the unscreened cross section (for bremsstrahlung in the field of a point Coulomb potential) can be integrated in closed form over the photon angles, and is given in [1], p. B1346, eq (6). The expression given in that reference is for arbitrary energies and angles, and is consequent ly rather lengthy. Since for our present considerations it is quite appropriate to assume $\epsilon_{1} \gg 1$ and $\epsilon_{2} \gg 1$, we give here the high energy form of that expression without, however, making the small angle approximation, $\vartheta \ll 1$. We then have 


$$
\frac{d \sigma_{u n s c r}}{d \Omega}=\frac{1}{2 \pi} \frac{e^{2}}{\hbar c}\left(\frac{Z e^{2}}{m c^{2}}\right)^{2} \frac{d \epsilon_{2}}{k \epsilon_{1}^{2}} \cdot 2 \epsilon_{1} \epsilon_{2} \cdot()
$$

where

$$
\begin{aligned}
& ()=\frac{k^{2} \lambda+2 \epsilon_{1} \epsilon_{2}(\lambda+1) \cos ^{2 \frac{1}{2} \vartheta}}{\lambda^{2}[\lambda(\lambda+2)]^{\frac{1}{2}}} 2 n\left\{\lambda+1+[\lambda(\lambda+2)]^{\frac{1}{2}}\right\} \\
& +\frac{k}{\lambda^{2}}\left[-k+\frac{\epsilon_{1}\left(\epsilon_{1}+\epsilon_{2}\right)}{\epsilon_{2}} \cos ^{2} \frac{1}{2} \vartheta+\frac{\epsilon_{1}{ }^{2}\left(2 \epsilon_{2}-k\right) \sin ^{2} \vartheta}{\lambda^{2}}\right] \ln \left(2 \epsilon_{2}\right) \\
& -\frac{k}{\lambda^{2}}\left[k+\frac{\epsilon_{2}\left(\epsilon_{1}+\epsilon_{2}\right)}{\epsilon_{1}} \cos ^{2} \frac{1}{2} \vartheta+\frac{\epsilon_{2}{ }^{2}\left(2 \epsilon_{1}+k\right) \sin ^{2} \vartheta}{\lambda^{2}}\right] \ln \left(2 \epsilon_{1}\right) \\
& +\frac{k^{2}}{2 \epsilon_{1} \epsilon_{2} \lambda}\left[\epsilon_{1}^{2}+\epsilon_{2}^{2}-2\left(\epsilon_{1}^{2}+\epsilon_{2}^{2}+\epsilon_{1} \epsilon_{2}\right) \cos ^{2} \frac{1}{2} \vartheta\right] \\
& +\frac{2 k^{2} \sin ^{2} \vartheta}{\lambda^{4}}\left(\epsilon_{1}{ }^{2}+\epsilon_{2}{ }^{2}-\epsilon_{1} \epsilon_{2}\right)-\frac{2 \epsilon_{1} \epsilon_{2}}{\lambda^{2}} \cos ^{2} \frac{1}{2} \vartheta .
\end{aligned}
$$

The high energy cross section for electrons which have scattered through an angle $\vartheta$ and radiated a photon, integrated over photon angles, is then given by eq (62), in which we substitute eqs (61) and (63). In these equations the variables $\delta, q_{m}$, and $\lambda$ are given by $(A 1),(A 12)$, and (A4), viz., 


$$
\begin{aligned}
\delta & =\frac{k}{2 \epsilon_{1} \epsilon_{2}} \\
q_{m} & =\frac{\lambda}{k} \\
\lambda & =\frac{k}{2 \epsilon_{1} \epsilon_{2}}+2 \epsilon_{1} \epsilon_{2} \sin 2 \frac{1}{2} \vartheta .
\end{aligned}
$$

It is to be noted that although the screening correction, eq (61), has the same relatively simple form whether we integrate over photon or electron angles, the unscreened cross section is very different in the two cases. The rather simple form that results for high energies and small angles when integratina over electron angles ([5], p. 924, Formula 28N(a)) is unfortunately not obtained when integrating over photon angles, as is clear from eq (63), even when we make the small angle approximation there, viz., by setting $\sin ^{2} \vartheta \approx \vartheta^{2}, \cos ^{2} \frac{1}{2} \vartheta \approx 1$. The rather complicated argument of the logarithm in (63) does not simpiify for small angles, where $\lambda=0(1)$. 
V. SCREENING CORRECTION IN THE THOMAS-FERMI-MOLIËRE MODEL

Although the screening correction is given formally by eq (61) as an integral over the atomic form factor, it is clear that a simple closed form expression without integrals is useful for practical applications, particularly if one must later perform an integration over this cross section. We therefore perform the integration in (61) in closed form for the Thomas-Fermi model as used by Moliere [7], viz., with

$$
\frac{1-F(q)}{q^{2}}=\sum_{i=1}^{3} \frac{\alpha_{i}}{B_{i}^{2}+q^{2}}
$$

where

$$
\begin{aligned}
& \alpha_{1}=0.10 \quad, \quad \alpha_{2}=0.55 \quad, \quad \alpha_{3}=0.35 \\
& \beta_{i}=\left(z^{1 / 3 / 121)}\right) b_{i} ; \quad b_{1}=6.0, \quad b_{2}=1.20, \quad b_{3}=0.30 \text {. }
\end{aligned}
$$

The result of this integration is then rather simple [6]: 
Defining the function $C\left(q_{m}\right)$ by

$$
\int_{q_{m}^{2}}^{\infty} \frac{\left[(1-F(q))^{2}-1\right]}{q^{4}}\left(\frac{q^{2}}{q_{m}^{2}}-1\right) d\left(q^{2}\right) \equiv-\frac{1}{q_{m}^{2}} c\left(q_{m}\right)
$$

we find

$$
\begin{aligned}
\dot{c}\left(a_{m}\right)= & \sum_{i=1}^{3} \alpha_{i}^{2} \ln \left(1+B_{i}\right) \\
& -2 \sum_{\substack{i=1 \\
i \neq j}}^{3} \sum_{j=1}^{3} \alpha_{i} \alpha_{j}\left[\frac{1+B_{j}}{B_{j}-B_{j}} \ln \left(1+B_{j}\right)+\frac{1}{2}\right]
\end{aligned}
$$

where

$$
B_{i}=\left(\frac{\beta_{i}}{q_{m}}\right)^{2}
$$

In the absence of screening, i.e., for $\frac{\beta_{i}}{a_{m}} \ll 1$, eq $(67)$ gives

$$
C\left(q_{m}\right)=0\left(\left(\frac{\beta_{i}}{q_{m}}\right)^{2}\right) \ll 1 \text {, }
$$

as expected. For $\frac{\beta_{i}}{q_{m}} \gg 1$. i.e., for complete screening, eq (67) gives 


$$
c\left(q_{m}\right)=\ln \left[\left(\frac{z^{1 / 3}}{111.8 q_{m}}\right)^{2}\right]+0\left(\frac{1}{\left(\frac{\beta_{i}}{q_{m}}\right)^{2}}\right) .
$$

Thus, if we write, in the limit of complete screening,

$$
c_{\text {compl.scr. }}\left(q_{m}\right)=\ln \left[1+\left(\frac{Z^{1 / 3}}{111.8 q_{m}}\right)^{2}\right]
$$

then both the complete screening and the no screening limits, eqs (70) and (69), are given by the single expression, (71). A comparison of $\mathcal{C}=\mathcal{C}\left(\mathrm{q}_{\mathrm{m}}\right)$ as given by (67) with $\mathcal{C}_{\text {compl.scr. }}$ as given by (71) shows that the relative error incurred by using $(71)$, viz., $\left(\mathcal{C}_{\text {compl }}\right.$ scr. $\left.-\mathcal{C}\right) / \mathcal{C}$, is less than $1 \%$ for $\frac{z^{1 / 3}}{111.8 q_{m}}>6.4$ and less than $2 \%$ for $\frac{z^{1 / 3}}{111.8 q_{m}}>4.7$.

For most practical applications requiring the screening correction, the expression given in (67) may be used for the integral in (61). Further, in the case of complete screeing, i.e., when $\frac{z^{1 / 3}}{111.8 q_{m}} \geq 5$, one may use the even simpler expression qiven in (71). 
In this appendix we derive the high energy approximations used in the body of the report, in particular for the variables $\delta, \lambda_{0}$, and $\lambda$ defined in (3), and for the quantity $\left(\frac{\epsilon_{1}}{p_{1}}-\frac{\epsilon_{2}}{p_{2}}\right)^{2}$, which enters in eqs (31) and (32). In addition we derive approximations for $\lambda$ and $a_{m}$, valid for both high energies and small scattering angle, ง.

By high energy approximation we mean $\epsilon_{1} \gg 1$ and $\epsilon_{2} \gg 1$, and imply neglect of terms of relative order $1 / \epsilon_{1}{ }^{2}$ and $1 / \epsilon_{2}{ }^{2}$, which we refer to as neglect of terms of relative order $1 / \epsilon^{2}$. By small angle approximation we mean $\vartheta \ll 1$ and imply neglect of terms of relative order $\vartheta^{2}$.

We start by deriving the high energy approximation for $\delta$, given in (3):

$$
\begin{aligned}
\delta & \equiv p_{1}-p_{2}-k \\
& =p_{1}-p_{2}-\epsilon_{1}+\epsilon_{2} \\
& =\left(\epsilon_{2}-p_{2}\right)-\left(\epsilon_{1}-p_{1}\right) \\
& =\frac{1}{\epsilon_{2}+p_{2}}-\frac{1}{\epsilon_{1}+p_{1}} \\
& =\frac{\epsilon_{1}+p_{1}-\epsilon_{2}-p_{2}}{\left(\epsilon_{1}+p_{1}\right)\left(\epsilon_{2}+p_{2}\right)} .
\end{aligned}
$$

Here 


$$
\begin{aligned}
p_{1}-p_{2} & =\frac{\left(p_{1}^{2}-p_{2}^{2}\right)}{p_{1}+p_{2}} \\
& =\frac{\left(\epsilon_{1}^{2}-\epsilon_{2}^{2}\right)}{p_{1}+p_{2}} \\
& =\left(\epsilon_{1}-\epsilon_{2}\right) \frac{\left(\epsilon_{1}+\epsilon_{2}\right)}{\left(p_{1}+p_{2}\right)} \\
& =k \frac{\left(\epsilon_{1}+\epsilon_{2}\right)}{\left(p_{1}+p_{2}\right)} .
\end{aligned}
$$

Thus,

$$
\begin{aligned}
\delta & =\frac{k\left(1+\frac{\epsilon_{1}+\epsilon_{2}}{p_{1}+p_{2}}\right)}{\left(\epsilon_{1}+p_{1}\right)\left(\epsilon_{2}+p_{2}\right)} \\
& =\frac{k\left(\epsilon_{1}+p_{1}+\epsilon_{2}+p_{2}\right)}{\left(p_{1}+p_{2}\right)\left(\epsilon_{1}+p_{1}\right)\left(\epsilon_{2}+p_{2}\right)} .
\end{aligned}
$$

Thus $f a r$ we have made no approximations, and have written $\delta$ in a form in which there are no cancellations. We can now set $p_{1}=\epsilon_{1}\left(1+O\left(1 / \epsilon_{1}^{2}\right)\right)$ and $p_{2}=\epsilon_{2}\left(1+O\left(1 / \epsilon_{2}^{2}\right)\right)$ and find, neglecting terms of relative order $1 / \epsilon_{1}{ }^{2}$ and $1 / \epsilon_{2}{ }^{2}$,

$$
\delta \approx \frac{k}{2 \epsilon_{1} \epsilon_{2}} .
$$


Next we consider (see (3))

$$
\begin{aligned}
\lambda_{0} & =\epsilon_{1} \epsilon_{2}-p_{1} p_{2}-1 \\
& =\frac{1}{2}\left[\left(p_{1}-p_{2}\right)^{2}-\left(\epsilon_{1}-\epsilon_{2}\right)^{2}\right] \\
& =\frac{1}{2}\left[\left(p_{1}-p_{2}\right)^{2}-k^{2}\right] \\
& =\frac{1}{2}\left(p_{1}-p_{2}-k\right)\left(p_{1}-p_{2}+k\right) \\
& =\frac{1}{2} \delta\left(p_{1}-p_{2}-k+2 k\right) \\
& =\delta\left(k+\frac{1}{2} \delta\right) .
\end{aligned}
$$

Again we have an expression with no cancellations. We can now use our high energy approximation for $\delta$, eq $(A 1)$, and obtain

$$
\begin{aligned}
\lambda_{0} & \approx \frac{k^{2}}{2 \epsilon_{1} \epsilon_{2}}+\frac{k^{2}}{8 \epsilon_{1}^{2} \epsilon_{2}^{2}} \\
& =\frac{k^{2}}{2 \epsilon_{1} \epsilon_{2}}\left(1+\frac{1}{4 \epsilon_{1} \epsilon_{2}}\right) .
\end{aligned}
$$

The second term is of relative order $1 / \epsilon^{2}$ and is thus neglected, giving the high energy approximation

$$
\lambda_{0} \approx \frac{k^{2}}{2 \epsilon_{1} \epsilon_{2}}
$$


Next, in eqs (31) and (32) we have the factor

$$
\left(\frac{\epsilon_{1}}{p_{1}}-\frac{\epsilon_{2}}{p_{2}}\right)^{2}=\frac{\left(\epsilon_{1} p_{2}-p_{1} \epsilon_{2}\right)^{2}}{p_{1}{ }^{2} p_{2}{ }^{2}}
$$

Here

$$
\begin{aligned}
\left(\epsilon_{1} p_{2}-p_{1} \epsilon_{2}\right)^{2} & =\epsilon_{1}{ }^{2} \epsilon_{2}{ }^{2}-\epsilon_{1}{ }^{2}-2 p_{1} p_{2} \epsilon_{1} \epsilon_{2}+p_{1}{ }^{2} p_{2}{ }^{2}+p_{1}{ }^{2} \\
& =\left(\epsilon_{1} \epsilon_{2}-p_{1} p_{2}\right)^{2}-1 \\
& =\left(\epsilon_{1} \epsilon_{2}-p_{1} p_{2}-1\right)\left(\epsilon_{1} \epsilon_{2}-p_{1} p_{2}+1\right) \\
& =\lambda_{0}\left(\lambda_{0}+2\right) .
\end{aligned}
$$

Using the high energy approximation for $\lambda_{0}$ just given in (A2) we have

$$
\begin{aligned}
\left(\epsilon_{1} p_{2}-p_{1} \epsilon_{2}\right)^{2} & \approx \frac{k^{2}\left(k^{2}+4 \epsilon_{1} \epsilon_{2}\right)}{\left(2 \epsilon_{1} \epsilon_{2}\right)^{2}} \\
& =\frac{k^{2}\left(\epsilon_{1}+\epsilon_{2}\right)^{2}}{\left(2 \epsilon_{1} \epsilon_{2}\right)^{2}} .
\end{aligned}
$$

We thus have the high energy approximation

$$
\left(\frac{\epsilon_{1}}{p_{1}}-\frac{\epsilon_{2}}{p_{2}}\right)^{2} \approx \frac{k^{2}\left(\epsilon_{1}+\epsilon_{2}\right)^{2}}{4 \epsilon_{1}^{4} \epsilon_{2}^{4}}
$$


which is given in eqs (31) and (32) as

$$
\left(\frac{\epsilon_{1}}{p_{1}}-\frac{\epsilon_{2}}{p_{2}}\right)^{2}=0\left(\frac{k^{2}}{\epsilon^{6}}\right) \text {. }
$$

Next we have the important variables $\lambda$ and $q_{m}$. From (3)

$$
\begin{aligned}
\lambda & =\epsilon_{1} \epsilon_{2}-p_{1} p_{2} \cos \vartheta-1 \\
& =\epsilon_{1} \epsilon_{2}-p_{1} p_{2}-1+p_{1} p_{2}(1-\cos \vartheta) \\
& =\lambda_{0}+2 p_{1} p_{2} \sin 2 \frac{1}{2} \vartheta .
\end{aligned}
$$

Thus from (A2) we have, at high energies (but arbitrary angle $\vartheta$ ),

$$
\lambda \approx \frac{k^{2}}{2 \epsilon_{1} \epsilon_{2}}+2 \epsilon_{1} \epsilon_{2} \sin 2 \frac{1}{2} \vartheta
$$

If in addition $\vartheta \ll 1$, as it is in the screening correction, then we have

$$
\begin{aligned}
\lambda & \approx \frac{k^{2}}{2 \epsilon_{1} \epsilon_{2}}+\frac{\epsilon_{1} \epsilon_{2} \vartheta^{2}}{2} \\
& =\frac{k^{2}}{2 \epsilon_{1} \epsilon_{2}}\left[1+\left(\frac{\epsilon_{1} \epsilon_{2} \vartheta}{k}\right)^{2}\right] .
\end{aligned}
$$

From (AI) this can be written as

$$
\lambda \approx k \delta\left[1+\left(\frac{\epsilon_{1} \epsilon_{2} \vartheta}{k}\right)^{2}\right] .
$$


This suggests that we define the variable w by

$$
w=\frac{\epsilon_{1} \epsilon_{2}^{\vartheta}}{k}
$$

from which

$$
\lambda \approx k \delta\left(1+w^{2}\right)
$$

or

$$
\frac{\lambda}{k} \approx \delta\left(1+w^{2}\right)
$$

Equation (A7) provides a useful expression for the minimum moment um transfer, $a_{m}$, in the region of importance for the screening correction, $q_{m} \lesssim O(\beta)$, since this condition, together with the assumption of high energies, implies small scattering angles. This may be seen from $q_{m}$ as defined in (3): Starting with

$$
q_{m}=\left(2 \lambda+k^{2}\right)^{\frac{1}{2}}-k \lesssim O(\beta)
$$

we write this as

$$
\left(2 \lambda+k^{2}\right)^{\frac{1}{2}}-k \leqq C B
$$

where $c$ is a constant of order unity. Thus

$$
\left(2 \lambda+k^{2}\right)^{\frac{1}{2}} \leqq k+C B .
$$


Squaring both sides we then have

$$
2 \lambda \leqq 2 c k \beta+c^{2} \beta^{2}
$$

which may be written as

$$
\lambda \lesssim O(k \beta)+O\left(\beta^{2}\right)
$$

With $\lambda$ as given at high energies in (A4), we have

$$
\lambda \approx \frac{k^{2}}{2 \epsilon_{1} \epsilon_{2}}+2 \epsilon_{1} \epsilon_{2} \sin ^{2 \frac{1}{2} \vartheta} \lesssim O(k \beta)+O\left(\beta^{2}\right)
$$

and hence

$$
2 \epsilon_{1} \epsilon_{2} \sin ^{2} \frac{1}{2} \vartheta \lesssim O(k B)+O\left(\beta^{2}\right)
$$

or

$$
\sin ^{2} \frac{1}{2} \vartheta \leq O\left(\frac{k B}{\epsilon^{2}}\right)+O\left(\frac{B^{2}}{\epsilon^{2}}\right) \leq O\left(\frac{\beta}{\epsilon}\right) \ll 1
$$

Thus the condition $q_{m} \leqslant O(\beta)$ (together with tha assumbtion $\epsilon_{1} \gg 1$, $\epsilon_{2} \gg 1$ ) implies small scattering angles

$$
\vartheta^{2} \lesssim O\left(\frac{B}{\epsilon}\right)
$$


for all photon energies, $k$. (In the body of this report we derived this result for $k=O(\epsilon)$; see eq (17).) We may thus use (A7) to obtain our expression for $q_{m}$ in the region $q_{m} \lesssim O(B)$. Again referring to (3), we have

$$
a_{m}=\left(2 \lambda+k^{2}\right)^{\frac{2}{2}}-k
$$

from which

$$
q_{m}^{2}+2 k q_{m}=2 \lambda
$$

or

$$
q_{m}=\frac{\frac{\lambda}{k}}{1+\frac{q_{m}}{2 k}} \text {. }
$$

Thus if we assume that the photons are not too soft, specifically, provided that *

$$
2 k \gg q_{m}
$$

then

$$
q_{m} \approx \frac{\lambda}{k}
$$

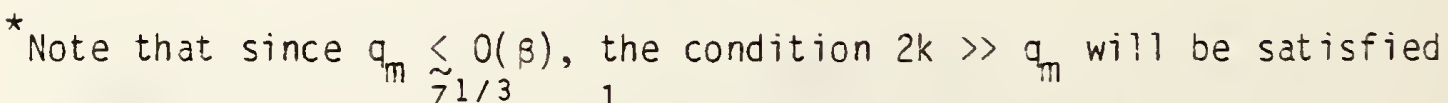
provided $2 k \gg \beta \sim \frac{\tilde{z} 1 / 3}{111.8} \leqslant \frac{1}{25}$. Thus to have $2 k \gg q_{m}$, it is sufficient to have $k \gg \frac{1}{50} \mathrm{mc}^{2} \approx 10 \mathrm{keV}$. 
and from (A7)

$$
q_{m} \approx \delta\left(1+w^{2}\right)
$$

It is worth noting the obvious similarity between (A13) and the high energy, small angle approximation for the minimum momentum transfer when one integrates over the final electron directions [6]. In that case

$$
\begin{aligned}
q_{m} & =\left|\underline{p}_{1}-\underline{k}\right|-p_{2} \\
& \approx \delta\left(1+u^{2}\right)
\end{aligned}
$$

where

$$
u=p_{1} \theta_{1}
$$

with

$$
\theta_{1}=\Varangle\left(\underline{p}_{1}, \underline{k}\right)
$$

When integrating over final electron directions [6] it was convenient to define

$$
\varepsilon=\frac{1}{1+u^{2}}
$$

in terms of which 


$$
q_{m}=\frac{\delta}{\xi}
$$

In the present situation, in which we integrate over photon directions, we define

$$
x=\frac{1}{1+w^{2}}
$$

in terms of which

$$
q_{m}=\frac{\delta}{x}
$$

Although in [6] the screening correction was expressed in terms of the variables $u$ and $\xi$, we note in the body of this report (see eq (61)) that the two screening corrections have the same form, identically, when expressed in terms of $q_{m}$, defined by $q_{m}=\left|\underline{p}_{1}-\underline{k}\right|-p_{2}$ when the photon is observed, and by $q_{m}=\left|\underline{p}_{1}-\underline{p}_{2}\right|-k$ when the final electron is observed. 


\section{REFERENCES}

[1] L. C. Maximon and D. B. Isabelle, Phys. Rev. 133, B1344 (1964).

[2] H. Nguyen-Ngoc and J. P. Perez-y-Jorba, Phys. Rev. 136, B1036 (1964).

[3] Y. S. Tsai, in Proceedings of the International Conference on Nuclear Structure, 1963, edited by Hofstadter and Schiff (Stanford University Press, Stanford, California, 1964), p. 221.

L. W. Mo and Y. S. Tsai, Rev. Mod. Phys. 41, 205 (1969).

Y. S. Tsai, SLAC-PUB 848 , January 1971.

[4] L. I. Schiff, Phys. Rev. 83,252 (1951).

[5] H. W. Koch and J. W. Motz, Rev. Mod. Phys. 31, 920 (1959).

[6] Haakon 01 sen and L. C. Maximon, Phys. Rev. 114, 887 (1959).

[7] G. Moliere, Zeits, f. Naturforsch. 2a, 133 (1947). 
HBS.114A IREV. $2.6 C$ I

U.3. DEPT. OF COMM.

BIBLIOGRAPHIC DATA

SHEET (See in structions)

1. PUBLICATION OR REPORT NO.

2. Performins Organ. Report Nof 3. Publication Date

4. TITLE AND SUBTITLE

Angular distribution of high energy electrons following radiation

5. AUTHOR(S)

Leonard C. Maximon and ATfred LePretre

6. PERFORMING ORGANIZATION (If jolnt or other than NBS, see instructions)

7. ContracuGrane No.

NATIONAL BUREAU OF STANADAROS

DEPARTHENT. OF COMHERCE

WASHINGTON, D.C. 20234

8. Type of Report \& Period Covered

9. SPONSORING ORGANIZATION NAME AND COMPLETE ADORESS (Street, City, Stote, ZIP)

10. SUPPLEMENTARY NOTES

Document describes a computer program; SF-185. FIPS Software Summary, is actached.

11. ABSTRACT (A 200-word or less foctual summary of most significant information. If document includes a significont blbliography or licerature survey. mentlon it here)

We derive an expression for the angular distribution of high energy electrons which have undergone scattering and radiated a photon, integrated over the directions of the emitted photon, in the region of small scattering angles, for which the atomic form factor must be taken into account, but the nuclear structure may be neglected. This distribution is analogous to Schiff's high energy small angle expression for the angular distribution of photons, integrated over final electron angles. We show that the correction to the angular distribution of electrons due to atomic screening is identical in form to the correction for the angular distribution of photons. This correction involves an integral over the atomic form factor, which is evaluated in closed form for the Thomas-Fermi-Moilere model. A very simple expression -is obtained for the case of complete screening.

12. KEY WOROS (Six to iwelve entries: alphobetical order: capilalize only proper names; and separace key words by semicolons) angular distribution of scattered electrons; atomic: screening effects in electron scattering; bremsstrahlung; high energy electron scattering; small angle electron scattering cross section: Schiff spectrum 13. AVAILABILITY

EUnilimled

$\square$ For Offlelal Distribution. Do Not Release to NTIS

$\square$ Order From Superintendent of Documents, U.S. Government Printing Office. Washinglon. D.C. 20402.

14. NO. OF PRINTEO PAGES

Order From National Technical Information Service (NTIS). Soringfield. VA. 22161

is. Price 

